\title{
Use of shielding integral benchmark archive and database for nucleardata validation
}

\author{
I. Kodeli ${ }^{1,3, *}, O$. Cabellos $^{2, * *}$, and L. Leal ${ }^{3, * * *}$ \\ ${ }^{1}$ Jozef Stefan Institute, Ljubljana, Slovenia \\ ${ }^{2}$ Polytechnic University of Madrid (UPM), Madrid, Spain \\ ${ }^{3}$ Institut de Radioprotection et de Surete Nucleaire, Paris, France
}

\begin{abstract}
Shielding benchmarks were extensively used for the validation and improvement of nuclear data since many years. Recent evaluations however mostly rely on the validation using critical benchmarks, which can introduce biases and compensation effects. A new Working Party on International Nuclear Data Evaluation Co-operation Subgroup 47 (WPEC SG47) entitled "Use of Shielding Integral Benchmark Archive and Database for Nuclear Data Validation" was started in June 2019 with the objective to promote further development and use of Shielding Integral Benchmark Archive and Database (SINBAD) and thus contribute to the diversification of the nuclear data validation practice by including more extensively other types of integral measurements, such as shielding benchmarks, in the validation and evaluation procedure. Use of shielding benchmarks is expected to provide a wider-scope test of the performance of the evaluated nuclear data and would ultimately contribute to a production of more general-purpose cross-section evaluations.
\end{abstract}

\section{Introduction}

The Shielding Integral Benchmark Archive and Database (SINBAD) project [1-6] started in the early 1990's as a collaboration between the Organization for Economic Cooperation and Development's Nuclear Energy Agency Data Bank (OECD/NEADB) and the Radiation Safety Information Computational Center (RSICC) at the Oak Ridge National Laboratory (ORNL) with the goal to preserve the information on the performed radiation shielding benchmarks and make them available in a standardised form to the international community. The latest version of SINBAD comprises over 100 shielding benchmarks, divided into three categories, covering both low and inter-mediate energy particles applications: fission reactor shielding ( 48 benchmarks), fusion blanket neutronics (31), and accelerator shielding (23) benchmarks. In addition to the characterization of the radiation source, description of shielding set-up, instrumentation and the relevant detectors, most sets in SINBAD contain also the deterministic or probabilistic (Monte Carlo) radiation transport computer model used for the interpretation of the experiment and, where available, results from uncertainty analysis. The set of primary documents used for the benchmark compilation and evaluation are provided in computer readable form.

The database is intended for different users, including nuclear data evaluators, computer code developers, exper-

\footnotetext{
*e-mail: ivan.kodeli@ijs.s

**e-mail: oscar.cabellos@upm.es

***e-mail: luiz.leal@irsn.fr
}

iment designers and university students. SINBAD is available from RSICC and from the NEA Data Bank.

\section{WPEC SG47}

Integral benchmark experiments have proven to be valuable for validation of nuclear cross-sections. In recent years, the evaluations have been predominantly validated against criticality benchmarks, and are 'tuned' to better match the measurements. However, the effective multiplication factor $\left(k_{e f f}\right)$ is a very global parameter, dependent on many 'free' parameters. Besides criticality, there are other important concerns, of which shielding is a priority for many applications. Including shielding benchmarks in the validation process is expected to provide a complementary view and would allow broader evaluation of the performance of the nuclear data. This would ultimately contribute to a production of general-purpose cross-section evaluations.

A new Working Party on International Nuclear Data Evaluation Co-operation Subgroup 47 (WPEC SG47) entitled "Use of Shielding Integral Benchmark Archive and Database for Nuclear Data Validation" [7] was started in June 2019 with the consider the above challenges and objectives, such as:

- To provide feedback on the existing database and contribute in this way to the quality review, concentrating on the verification of the completeness and the consistency of the experimental information (on the geometry, material composition, the procedure to derive data-unfolding, etc.), in particular concerning the evaluation of experimental sources of uncertainty. 
- Provide recommendations on the SINBAD evaluations based on the experience, needs and expectations of the nuclear data community; To participate in establishing the priority list of relevant benchmarks according to the needs of ND community, in particular among new and recent benchmarks; promote including the selected benchmarks in SINBAD; contribute sensitivity profiles.

- To participate, in coordination with EGRTS WPRS, in establishing the review group and organisation of pilot exercise of SINBAD evaluations. The FNG benchmark evaluations performed within the EC Fusion for Energy (F4E) project are good candidates for the pilot exercise.

WPEC SG47 will work in close coordination with the SINBAD project and Expert Group on Radiation Transport and Shielding (EGRTS) of OECD/NEA. WPEC SG47 shall provide feedback on the existing database, promote further development and provide recommendations on the SINBAD evaluations based on the experience, needs and expectations of the nuclear data community.

Cooperation with other NEA activities [8] such as WPEC SG45 (Validation of Nuclear Data Libraries (VaNDaL) Project), SG46 (Efficient and Effective Use of Integral Experiments for ND Validation), JEFF [9] and European Comission (EC) projects such as SANDA and EUROfusion is likewise important in order to study and recommend the proper mathematical and physics procedures to integrate these data in the ND evaluation process and thus avoid their misuse. Several laboratories from US (LLNL, ORNL) China (FDS, CIAE), Russia (IPPE) and others expressed interest in this activity.

\section{Present status and quality review}

Several of the SINBAD benchmarks are old, some dating back to the 1960s and the access to the detailed and reliable experimental information neede for the validation of the present high-quality nuclear data is difficult or not possible. Still, these benchmarks are valuable since they provide the record of experimental data on which present data and methods are often based. On the other hand, some of these experiments may not be best suited for the present nuclear data validation and improvement. A thorough revision and classification of the benchmark experiments according to the completeness, reliability and consistency of information was therefore started about 10 years ago. More than half of the SINBAD experiments were already, or are currently being revised and reclassified [10-13]. The review concentrated on the verification of the description of the experimental setup, the neutron source specifications, the detector characteristics, the geometry and precise material composition of the components. The main criteria for the judgment of the quality of the experiment are the completeness and the consistency of the experimental information (on the geometry, materials, the procedure to derive data-unfolding, etc.), in particular concerning the evaluation of the experimental sources of uncertainty. New or improved inputs for computer codes such as MCNP were prepared and the sensitivity analyses were performed to estimate the impact of the approximations used in the computational model. Other models, such as for SuperMC [16] are planned to be added. The following two guidelines were pursued when preparing the computational models:

- Calculation should be able to reproduce the experiment as exactly as reasonably possible, avoiding unnecessary approximations;

- As much as possible the calculations should be compared to pure measured data. Clear distinction is needed between 'processed' measured data and 'measured' (e.g. time-of-flight experiments), with rigorous evaluation of the involved computational approximations and uncertainties.

This review process is expected to provide the users with an easier choice and help them make better use of the experimental information.

\section{Candidates for future SINBAD evaluations}

SINBAD database includes now over 100 benchmarks. The progress was slow in the recent years but the activities on further expansion of the database shall continue. The objectives of the future work are twofold, on one side to continue with the more detailed and complete quality evaluations of the existing database, and on the other side to complement the database with new benchmark evaluations.

At the WPEC SG47 kick-off meeting several past shielding benchmarks of interest for nuclear data validation were identified. Among the candidates for future SIN$\mathrm{BAD}$ evaluations the following can be highlighted:

- FNG-Cu and FNG-HCLL: evaluations done within F4E in 2018, review needed

- FNS $\mathrm{Cu}, \mathrm{Ti}, \mathrm{Li}_{2} \mathrm{O}$ : requested from JAEA

- OKTAVIAN benchmarks

- LLNL pulsed spheres

- IPPE: BTiH, U

- CIAE neutron leakage spectra from $\mathrm{Fe}, \mathrm{SiC}$, graphite

- REZ Fe sphere and slab benchmarks [17]

- VENUS-1, VENUS-2 PV dosimetry experiment

- JET: SDR experiment (2012-2013), streaming, dose rate

- Neutron Penetration through Fe and Concrete for 140350-MeV Quasi-Monoenergetic Neutrons, RCNP, Osaka University,

- JASMIN: Japanese-American Study of Muon Interactions and Neutron Detection FERMILAB, Japan Fermilab

- Measurements of reaction rates and induced activity in concrete exposed to secondary particles produced by intermediate energy heavy ions on Fe target HIMAC (NIRS)

- Neutron transmission experiments by V. Fillipov in 1960 ( $\mathrm{Si}, \mathrm{Nb}, 1 \mathrm{keV}$ to $3 \mathrm{MeV}$ ) 
- KFK 1977 iron sphere gamma measurements using ${ }^{252} \mathrm{Cf}$ source

- NIST 2000 50-cm Fe sphere experiment using ${ }^{252} \mathrm{Cf}$ source

- LRC, Ohio neutron leakage spectra from Ta sphere with Am-Be source

- Ohio University Fe spheres with $\mathrm{D}(\mathrm{d}, \mathrm{n})$ neutron source

- Mn bath experiment

\subsection{Recent SINBAD evaluations - FNG Copper Benchmark}

A neutronics benchmark experiment on a pure Copper assembly [14] was performed end 2014 - beginning 2015 at the 14-MeV Frascati neutron generator (FNG) of ENEA Frascati with the objective to provide the experimental database required for the validation of the copper nuclear cross-section data relevant for ITER design calculations, including the related uncertainties. Evaluation of the benchmark for the SINBAD database was performed in the scope of the F4E project [15] and the review is ongoing to include the data in SINBAD. FNG Cu benchmark is planned to be used as a pilot evaluation in the scope of WPEC SG47.

The experiment was performed in the frame of the European Fusion Program. Reaction rates, neutron flux spectra and doses were measured at several locations inside the 60x70x70 $\mathrm{cm}^{3}$ Copper block (Fig. 1) using different experimental techniques $\left({ }^{197} \mathrm{Au}(\mathrm{n}, \gamma),{ }^{186} \mathrm{~W}(\mathrm{n}, \gamma),{ }^{55} \mathrm{Mn}(\mathrm{n}, \gamma)\right.$, ${ }^{115} \mathrm{In}\left(\mathrm{n}, \mathrm{n}\right.$ '), ${ }^{58} \mathrm{Ni}(\mathrm{n}, \mathrm{p}),{ }^{58} \mathrm{Ni}(\mathrm{n}, 2 \mathrm{n}),{ }^{27} \mathrm{Al}(\mathrm{n}, \alpha),{ }^{93} \mathrm{Nb}(\mathrm{n}, 2 \mathrm{n})$ activation foils, NE213 scintillator and thermoluminescent detectors).

The reference analyses of the experiment was carriedout using the MCNP5 Monte Carlo code and the European JEFF-3.2 cross-section library. A simplified 2D model for the deterministic DORT code was also prepared. The preand post-analysis of the experiment was complemented by cross-section sensitivity and uncertainty $(\mathrm{S} / \mathrm{U})$ calculations using both deterministic (SUSD3D) and Monte Carlo (MCSEN5) codes. Cumulative reaction rates and neutron flux spectra, their sensitivity to the cross sections, as well as the corresponding uncertainties were estimated for selected detector positions up to $\sim 58 \mathrm{~cm}$ in the copper assembly. This permitted to interpret the results of the measurements and the calculations to conclude on the quality of the relevant nuclear cross-section data, and to estimate the uncertainties in the calculated nuclear responses and fluxes. Large uncertainties in the calculated reaction rates and neutron spectra of up to $50 \%$, rarely observed at this level in the benchmark analysis using today's nuclear covariance data, were predicted, particularly high for fast reactions (Table 1). Large discrepancies between calculations $(\mathrm{C})$ and experiment $(\mathrm{E})$ for the reaction rates both in the high and low neutron energy range partly confirm the predictions of the $\mathrm{S} / \mathrm{U}$ analysis. $\mathrm{C} / \mathrm{E}$ values as low as 0.5 were observed for the presently available nuclear data for copper, regardless of the data evaluation and version (JEFF, FENDL, ENDF/B, JENDL).
The sensitivity/uncertainty analyses enabled to identify the cross sections and energy ranges which are mostly affecting the calculated responses. The largest discrepancy among the $\mathrm{C} / \mathrm{E}$ values was observed for the thermal (capture) reactions indicating severe deficiencies in the ${ }^{63,65} \mathrm{Cu}$ capture and elastic cross sections at lower rather than at high energy.

Benchmark experiment is therefore expected to contribute to the improvement of both cross section as well as covariance data evaluations.

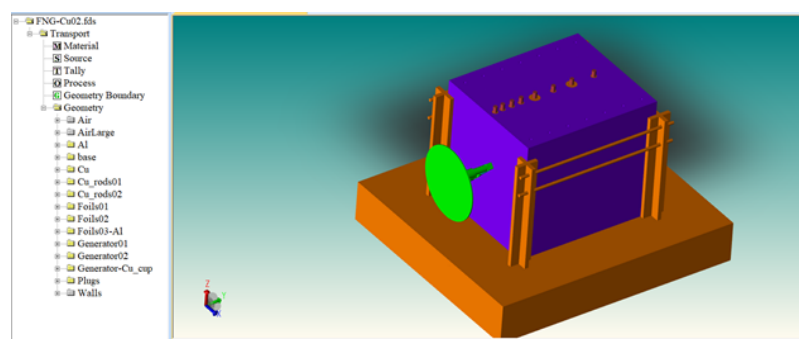

Figure 1. Experimental set-up of the FNG Copper benchmark (CAD geometry)

\section{Conclusions}

The SINBAD database currently contains compilations and evaluations for 102 fission, fusion and accelerator relevant shielding problems and continues to represent an important experimental database for validating nuclear data, codes and nuclear design. The SINBAD database is now widely used for code and data validation. Materials covered include: Air, N. O, $\mathrm{H}_{2} \mathrm{O}, \mathrm{Al}, \mathrm{Be}, \mathrm{Cu}$, graphite, concrete, $\mathrm{Fe}, \mathrm{Pb}, \mathrm{Li}, \mathrm{Ni}, \mathrm{Nb}, \mathrm{SiC}, \mathrm{Na}, \mathrm{SS}, \mathrm{W}, \mathrm{V}$ and mixtures thereof. Over 40 organisations from 14 countries and 2 international organisations have contributed data and work in support of SINBAD. SINBAD was successfully used in the scope of Fusion for Energy (F4E), EUROfusion, CHANDA, WPEC CIELO, SG39, SG46, EFF-4 and IAEA activities and demonstrated to be suitable for validation of modern nuclear data evaluations and codes, in combination with criticality, kinetics and other benchmarks. It is gradually being recognized, for example within the European experimental fusion programmes F4E and EUROfusion, that the benchmark experiment is fully completed only after the corresponding information is safely stored and evaluated for consistency and completeness, and not when the measurements were performed.

In the last decade the revision and classification of the benchmark experiments according to the completeness and reliability of information is being undertaken in order to provide users with easier choices and help them make better use of the experimental information. The quality review was completed for about half of the benchmarks and shall continue. In the overall, progress was however slow since 2009 with significantly less new benchmark evaluations per year as in the previous periods. Only recently, two new evaluations were performed in the scope of F4E and some more may come in the near future. The new 
Table 1. FNG-Cu benchmark: Comparison of the $\mathrm{C} / \mathrm{E}$ values and the computational uncertainty due to transport cross-sections using different nuclear data evaluations.

\begin{tabular}{r|ccc|cc}
\hline Reaction \& & \multicolumn{3}{|c|}{ Uncertainty (\%) } & \multicolumn{2}{c}{ C/E } \\
position $(\mathrm{cm})$ & JEFF-3.3 & ENDF/B-VI.8 & TENDL-2013 & FENDL3 & JEFF3.2 \\
\hline${ }^{58} \mathrm{Ni}(n, p) 35$ & 4.8 & 13.7 & 22.9 & 1.03 & 0.98 \\
57 & 9.1 & 27.2 & 41.9 & 1.03 & 0.91 \\
${ }^{115} \mathrm{In}\left(n, n^{\prime}\right) 35$ & 8.2 & 9.4 & 12.1 & 0.78 & 0.68 \\
57 & 12.7 & 18.7 & 23.5 & 0.69 & 0.54 \\
${ }^{27} \mathrm{Al}(n, \alpha) 57$ & 12.5 & 33.2 & 51.9 & 0.88 & 0.77 \\
${ }^{93} \mathrm{Nb}(n, 2 n) 57$ & 13.3 & 34.7 & 53.4 & 0.92 & 0.79 \\
${ }^{197} \mathrm{Au}(n, \gamma) 57$ & 15.3 & 19.9 & 18.6 & 0.58 & 0.63 \\
${ }^{186} \mathrm{~W}(n, \gamma) 57$ & 23.2 & 28.6 & 27.3 & 0.41 & 0.37 \\
\hline
\end{tabular}

FNG-Cu SINBAD benchmarks prepared in 2018 in the scope of F4E project includes few novelties such as sensitivity profiles, geometry in CAD format, www acceleration of MCNP calculations using ADVANTG code reducing CPU time from weeks to only few minutes.

Many discussions were devoted recently to the format issues rather than the content itself. Reformatting the database is likely to require considerable funding and extreme caution to avoid a possible (probable) loss of data in the process of re-formatting. In the actual situation of limited funding we suggest that the main activity be preferentially focused on the new benchmark evaluations according to the SINBAD priority list and on continuation of the quality revision and classification of existing benchmark.

A new WPEC SG47 on "Use of Shielding Integral Benchmark Archive and Database for Nuclear Data Validation" started in June 2019. The Subgroup is expected to provide feedback on the expectations and needs of the nuclear data community and thus contribute and orient the further development of the database.

Further development of SINBAD relies heavily on contributions from scientists and experimentalists. Proposals and assistance in new benchmark compilations are welcome.

\section{References}

[1] SINBAD database, OECD-NEA, abstracts available online: https://www.oecdnea.org/science/wprs/shielding/sinbad/

[2] P. Miller, P. Nagel, M. Salvatores, E. Sartori, "Shielding Experimental Benchmark Base at the Nuclear Energy Data Bank", Proc 7th Int. Conf. on Rad. Shielding, Bournemouth, UK (1988) NEACRP-L-310.

[3] A.K. McCracken, E. Sartori, "A Proposed Structure for a Shielding Experimental Benchmark Data Bank", Report NEACRP-A-1020, OECD/NEA (1989).

[4] I. Kodeli, E. Sartori, B. Kirk, Nuclear Instruments and Methods in Physics Research A 562 (2006).
[5] B. L. Kirk, R. E. Grove, I. Kodeli, J. Gulliford, E. Sartori, J. ASTM International (JAI), 9 (March 2012), Iss. 3.

[6] I. Kodeli, A. Milocco, P. Ortego, E. Sartori, Prog. in Nucl. Sci. \& Technology, 4 (2014) 308-311.I.

[7] I. Kodeli, O. Cabellos, L. Leal, Proposal for WPEC Sub-group 47 on "Use of Shielding Integral Benchmark Archive and Database for $\mathrm{Nu}$ clear Data Validation" (2018). https://www.oecdnea.org/science/wpec/sg47/index.html

[8] M. Fleming et al., Overview of the OECDNEA Working Party on International Nuclear Data Evaluation Cooperation (WPEC) (this conference). https://www.oecd-nea.org/science/wpec/

[9] A. Plompen, et al., The Joint Evaluated Fission and Fusion (JEFF) Nuclear Data Library (this conference)

[10] I. Kodeli, "Transport and S/U Analysis of the ASPISIron88 Benchmark Using Recent and Older Iron CrossSection Evaluations", Proc. PHYSOR 2018, Cancun, Mexico, Apr.22-26, 2018.

[11] I. Kodeli, A. Milocco, A. Trkov, Nucl. Technol. 168 (2009), pp.965-969.

[12] A. Milocco, A. Trkov, I. Kodeli, Annals of Nuclear Energy 37 (2010), pp.443 - 449.

[13] A. Milocco, I. Kodeli, A. Trkov, "The 2010 compilation of SINBAD: Quality assessment of the fusion shielding benchmarks", Proc. NEMEA-6, Krakow, Poland, 25-28 Oct. 2010.

[14] M. ANGELONE, et al., "Copper benchmark experiment for the testing of JEFF-3.2 nuclear data for fusion applications", EPJ Web of Conferences 146, 09004 (2017).

[15] I. Kodeli, M. Angelone, Fus. Eng. Design (2019) Nucl. Fusion 52 (2012) 083014.

[16] C. Chen, Q. Yang, B. Wu, Y. Han, J. Song, FDS Team, EPJ Web of Conferences 153, 02009 (2017) DOI: $10.1051 /$ epjconf/20171530

[17] B. Jansky, et al., EPJ Web of Conferences 146, 06021 (2017) DOI: 10.1051/epjconf/201714606021 